\title{
Lymphödem, Inflammation und neue Therapieansätze
}

\section{Lymphoedema, inflammation and new therapeutic approaches}

\author{
Autor \\ Anya Miller \\ Institut \\ Praxis für Dermatologie, Allergologie, Lymphologie und \\ Phlebologie, Berlin \\ Schlüsselwörter \\ Lymphödem, Entzündung, T-Lymphozyten, Makrophagen, \\ LTB4, IL-6, Ketoprofen, Hydroxytyrosol \\ Key words \\ lymphoedema, inflammation, t-lymphocytes, macrophages, \\ LTB4, IL-6, ketoprofen, hydroxytyrosol \\ online publiziert 04.03 .2021 \\ Bibliografie \\ Phlebologie 2021; 50: 131-134 \\ DOI 10.1055/a-1383-7624 \\ ISSN 0939-978X \\ (c) 2021. Thieme. All rights reserved. \\ Georg Thieme Verlag KG, Rüdigerstraße 14, \\ 70469 Stuttgart, Germany \\ Korrespondenzadresse \\ Dr. Anya Miller \\ Fachärztin für Haut- und Geschlechtskrankheiten \\ Allergologie/Phlebologie/Lymphologie, \\ Wilmersdorfer Straße 62, 10627 Berlin, Deutschland \\ miller@miller-derma.de
}

\section{ZUSAMMENFASSUNG}

Der fehlende Abtransport eiweißreicher interstitieller Flüssigkeit führt zu den bekannten klinischen Zeichen der Lymphödems an der Haut, die im Wesentlichen durch eine Fibrosierung ausgelöst werden. Bisher basiert die Therapie auf der mechanischen Anregung des Lymphtransports durch die komplexe physikalische Entstauung (KPE) oder operativen Maßnahmen, um das Ödem zu reduzieren. Der komplexe Ablauf der Entzündungsvorgänge im Gewebe wurde in den vergangenen Jahren untersucht und zeigt die zentrale Bedeutung von T-Lymphozyten, Makrophagen, LTB4 und diversen Zytokinen. Ausgehend von diesen Erkenntnissen gibt es Erfolg versprechende Therapieansätze mit Ketoprofen, Hydroxytyrosol und weiteren Immunmodulatoren.

\section{ABSTRACT}

Insufficient removal of protein rich interstitial fluid leads to typical changes of the skin, which is caused by fibrosis. Treatment of lymphedema consists basically in mechanic enhancement of the lymph transport with complex decongestive therapy or surgery. In the past years research on inflammation in lymphedema tissue demonstrated the important role of T-lymphocytes, macrophages, LTB4 and some cytokines. Regarding these findings we have new therapeutic aspects with ketoprofen, hydroxytyrosol and other immune modulators.

\section{Einleitung}

Als Ödem wird eine Schwellung von Gewebe durch Flüssigkeitseinlagerung bezeichnet, und so vielfältig die Ursachen sind, so unterschiedlich sind auch die Folgen im Gewebe und vor allem in der Haut. Auch das langjährig bestehende distale Ödem bei Herzinsuffizienz verursacht keine Pachydermie. Bei der chronischen venösen Insuffizienz (CVI) findet sich das Ödem zunächst am Unterschenkel, während der Fuß lange schlank bleibt. Nach Dermatosklerose, Dermatoliposklerose und Dermatolipofasziosklerose kann lokal ein Ulcus cruris entstehen. Die Folgen des chronischen Lymphödems hingegen sind häufig zuerst am Fuß sichtbar und zeigen sich in Form von Pachydermie, Papillomatose, Zysten und Fisteln. Dabei sind die Veränderungen im Gewebe abhängig von der Lokalisation, und es ist unerheblich, ob es sich um ein primäres öder sekundäres Lymphödem handelt. Die Reaktion des Gewebes auf die dauerhaft vermehrte Ansammlung lymph- pflichtiger Substanzen ist aber prinzipiell im Sinne einer Inflammation zu sehen. Diese Entzündungsreaktion involviert die Lymphgefäße, Blutkapillaren und vor allem das Gewebe.

\section{Folgen am Lymphgefäßsystem}

Entgegen früherer Annahmen dient das Lymphgefäßsystem nicht nur der Durchleitung von Lymphe, sondern produziert selbst auch Zytokine, die eine Auswirkung auf den Entzündungsprozess haben. Bei zunehmender Dauer eines Lymphödems wird aus Makrophagen IFN $\gamma$, TNFaund IL-ß freigesetzt [1]. IL-ß induziert vermutlich die Freisetzung von IL-6 aus Lymphendothelzellen. Das ist dann u. a. verantwortlich für zunehmende Durchlässigkeit der Lymphsinus, Präkollektoren und Kollektoren und es entsteht eine lymphatische Hyperplasie mit der Bildung aberranter lymphatischer Kanäle. Histopathologisch zeigen sich eine fibroadipöse Umwandlung mit einem Kollagenmantel um initiale 
Lymphgefäße und eine Sklerosierung von Kollektoren, die zur Obliteration führt. Die Folge ist ein Funktionsverslust mit weiterer Flüssigkeitsakkumulation im Gewebe.

\section{Reaktion der Kapillaren}

An den Blutkapillaren führt das eiweißreiche Ödem mit Persistenz von Sauerstoffradikalen zur Schädigung der Glykokalyx und Bildung von Lipoperoxyden und Aldehyden. Aus den Endothelzellen wird IL-1 und IL-6 freigesetzt [2].

\section{Folgen im Gewebe}

Das Lymphödem löst eine Aktivierung dendritischer Zellen aus, die zu den regionalen Lymphknoten migrieren und dort über T-ZellRezeptoren und kostimulatorische molekulare Interaktion naive T-Zellen aktivieren. Diese aktivierten T-Zellen gelangen wieder, angelockt durch Chemokine, die Keratinozyten und Leukozytenadhäsionsmoleküle aus Blutendothelzellen freisetzen, in die vom Lymphödem betroffene Hautregion [2].

Aktivierte T-Helferzellen differenzieren in Th1, Th2, Th17, regulatorische T-Zellen und Weitere. Th2-Zellen führen zu einer Immunsuppression, Inhibition von lymphatischem Wachstum und einem Anstieg profibrotischer Zytokine wie IL-4, IL-13 und TGF-B. Gleichzeitig hemmen sie den Gewebeuntergang. Dem entgegen stehen Th1-Zellen, die die Fibrosierung hemmen und bei Erhöhung für eine Balance der Th2-Zellen verantwortlich sind. Entscheidend für die Veränderung im Gewebe ist das Verhältnis zwischen Th1- und Th2-Zellen [3, 4].

Aktvierte Makrophagen exprimieren Interleukin IL-1 und IL-6. IL-1 induziert eine lokale Entzündung, die das Bindegewebe schädigt, Endothelzellen verdrängt und die Basallamina zerstört. IL-6 als proinflammatorisches Akute-Phase-Protein spielt eine zentrale Rolle bei der Entzündung und ist im Lymphödemgewebe erhöht nachweisbar [4]. IL-6 ist zudem ein Regulator der Adipogenese [5]. Makrophagen fördern die Expression freier Sauerstoffradikale (iNOS), die die Kontraktionsfähigkeit der Lymphangiome reduzieren [6]. Makrophagen exprimieren außerdem VEGF-C. Eine starke Überexpression von VEGF-C reduziert Fibrose.

Dendritische Zellen produzieren, induziert durch IL-1 und TNF- $\alpha$, Matrix-Metalloproteinasen (MMP), um den Weg durch die Basalzellschicht zu bahnen [7]. Sie zerstören elastische Fasern und sind für die Fibroblastenaktivierung mit Kollagenbildung verantwortlich. Dadurch wird sowohl die Fibrosierung als auch die Umwandlung in Fettgewebe gefördert.

Ein wichtiger Aktivator ist auch Leukotrien B4 (LTB4). Leukotriene sind aktivierte Lipide, die ursprünglich aus Leukozyten isoliert wurden. Sie sind Arachidonsäure-Metaboliten, die aus proinflammatorischen Immunzellen wie dendritischen Zellen, Makrophagen, Eosinophilen, Mastzellen und Neutrophilen gebildet werden. In niedrigen Konzentrationen stimulieren sie in vitro lymphangiologisches Wachstum, aber in hohen Konzentrationen ändert sich die Wirkung in das Gegenteil und sie wirken hemmend auf die Lymphangiogenese [8].

Über die Inhibition des Notch Pathway hemmt LTB4 IL-4 und IL-13. Eine Herunterregulation von LTB-Signalen reduziert die Infil- tration von CD8 +- und CD4 +-Zellen, Neutrophilen und Makrophagen in Lymphödemen [8]. Außerdem wird der Wachstumsfaktor VEGFR3, ein wichtiger Bestandteil der Lymphangiogenese, aktiviert [9]. LTB4 führt so zur Einschränkung der Funktion von Lymphendothelzellen und führt zu einer Progression des Lymphödems $[8,10]$.

Diese Vorgänge sind Teil einer Reaktionskaskade, bei der viele Einzelheiten bisher ungeklärt sind.

\section{Therapieansätze}

Ausgehend von einer Inflammation als Ursache für die Fibrosierung im Gewebe wurden verschiedene Therapieansätze mit Fokus auf die Entzündungshemmung in Studien untersucht.

Die Hemmung von Th2-Differenzierung durch IL-4- oder IL-13Blockade führte in Studien mit Mäusen zu einer Reduktion der Fibrosierung [11, 12] und Besserung der lymphatischen Funktion. Das konnte auch durch die Gaben mit monoklonalen Antikörpern bestätigt werden [11, 13]. Aktuelle klinische Studien, z. B. mit Dupilumab als IL-4- und IL-13-Blocker, und die Auswirkungen auf die Fibrosierung beim Lymphödem sind nicht bekannt.

\section{Ketoprofen}

Ketoprofen ist ein nichtsteroidaler Entzündungshemmer aus der Gruppe der Propionsäure-Derivate und ist bekannt in der Anwendung bei Schmerzen und Entzündung. Ketoprofen wirkt antiinflammatorisch durch Hemmung von Cyclooxgenase (COX) und 5-Lipoxygenase (5-LO) [8]. Untersuchungen an Mäusen zeigten bei systemischer Gabe eine deutliche Besserung des Lymphödems. Studien (Open label und Placebo-kontrolliert) an insgesamt 30 Patienten in der Verum-Gruppe und 16 in der PlaceboGruppe ergaben keine Reduktion des Armvolumens, aber eine Verbesserung der Histopathologie der Haut und der Hautdicke bei Nachweis der Inhibition von LTB4 und Reduktion von 5-LO [8, 14].

\section{Hydroxytyrosol}

Hydroxytyrosol, z. B. als Bestandteil von Oliven, hemmt die 5-Lipoxygenase und blockiert dadurch die Bildung von LTB4 aus Arachidonsäure. Dadurch wird in vitro die gleiche Wirkung auf LTB4 erzeugt wie durch Ketoprofen. Klinische Studien stehen aus, aber Hydroxytyrosol aus Oliven wurde als nebenwirkungsarm bestätigt [15].

\section{Tacrolimus}

Tacrolimus senkt die IL-2-Aktivität, die Produktion von CD4 +-Zellen (T-Helferzellen, Makrophagen, Monozyten) und reduziert die Fibrose. Maßgeblich für die Wirkung ist die Hemmung der Makrophagen. Lappenplastiken zeigen nach Applikation eine deutlich geringere Nekroserate [16].

\section{Diskussion}

Für die Lebensqualität des Lymphödem-Patienten sind Volumen und Konsistenz des Gewebes ebenso wichtig wie die Vermeidung 
- Tab. 1 Beteiligte Immunmodulatoren.

\begin{tabular}{|c|c|c|c|}
\hline & Bildung/Ursprung & Funktion & Antikörper \\
\hline IL-1 & $\begin{array}{l}\text { Makrophagen, Endothelzellen, } \\
\text { Fibroblasten }\end{array}$ & $\begin{array}{l}\text { - induziert lokale Entzündung } \\
\text { - induziert Migration dendritischer Zellen } \\
\text { - schädigt Bindegewebe } \\
\text { - verdrängt Endothelzellen }\end{array}$ & \\
\hline IL-1 $\alpha$ & $\begin{array}{l}\text { Sezernierung durch Monozyten } \\
\text { und Makrophagen }\end{array}$ & $\begin{array}{l}\text { - Erhalt der Hautbarrierefunktion } \\
\text { - regt Produktion von Kollagen in der Dermis an }\end{array}$ & \\
\hline IL-1b & Makrophagen, Monozyten & $\begin{array}{l}\text { - erzeugt Fieber } \\
\text { - Akute-Phase-Protein } \\
\text { - Anstieg von IL-6 }\end{array}$ & \\
\hline IL-4 & $\begin{array}{l}\text { Th2-Zellen, basophile und eosino- } \\
\text { phile Granulozyten, Mastzellen, } \\
\text { NK-Zellen }\end{array}$ & $\begin{array}{l}\text { " antiinflammatorisch, verringert Produktion von Th1 und } \\
\text { Makrophagen } \\
\text { - fördert M2-Makrophagenaktivierung } \\
\text { " differenziert Th0-zu Th2-Zellen, } \\
\text { " reduziert Lymphangiogenese } \\
\text { " profibrotisch }\end{array}$ & Dupilumab \\
\hline IL-6 & $\begin{array}{l}\text { Monozyten, Makrophagen, Epithel- } \\
\text { zellen, Endothelzellen, Lymphendo- } \\
\text { thelzellen }\end{array}$ & $\begin{array}{l}\text { - proinflammatorisches Akute-Phase-Protein } \\
\text { - involviert in Fettgewebshomöostase }\end{array}$ & $\begin{array}{l}\text { Tocilozumab } \\
\text { Sarilumab }\end{array}$ \\
\hline IL-13 & T-Lymphozyten & $\begin{array}{l}\text { - stimuliert Bildung und Differenzierung von B-Lymphozyten } \\
\text { - fördert M2-Makrophagenaktivierung } \\
\text { - profibrotisch } \\
\text { - reduziert Lymphangiogenese }\end{array}$ & Dupilumab \\
\hline INF-Y & $\begin{array}{l}\text { Th1, CD4 + nach Kontakt mit } \\
\text { Makrophagen }\end{array}$ & $\begin{array}{l}\text { - reduziert Lymphangiogenese } \\
\text { - aktiviert Makrophagen }\end{array}$ & \\
\hline Th1 & Lymphknoten & - Fibrosehemmung & \\
\hline Th2 & Lymphknoten & - Freisetzung von IL-4 und IL-13 & \\
\hline TGF-ß & & $\begin{array}{l}\text { - Profibrotisch } \\
\text { - Inhibitor der Lymphangiogenese }\end{array}$ & \\
\hline MMP & & $\begin{array}{l}\text { - Zerstörung elastischer Fasern } \\
\text { - Fibroblastenaktivierung } \\
\text { - Kollagenbildung }\end{array}$ & \\
\hline LTB4 & $\begin{array}{l}\text { dendritische Zellen, Mastzellen, } \\
\text { Eosinophile, Neutrophile }\end{array}$ & $\begin{array}{l}\text { - über Notch Pathway Hemmung IL-4 und IL-13, Aktivierung } \\
\text { VEGFR3 } \\
\text { - fördert Bildung von Th17 } \\
\text { - fördert Infiltration mit CD4 +-, CD8 +-Zellen und } \\
\text { Makrophagen } \\
\text { - Reduktion der Funktion von Lymphendothelzellen } \\
\text { - Verschlechterung des Lymphödems }\end{array}$ & \\
\hline VEGF-c & & - fördert Lymphangiogenese & \\
\hline
\end{tabular}

von Spätfolgen. Dabei sind die Veränderungen im Gewebe zentral für die Funktionstüchtigkeit des Lymphgefäßsystems. Diese Veränderungen verlaufen in einem Entzündungsprozess, der maßgeblich von Makrophagen, T-Lymphozyten, LTB4 und einer Reihe von Zytokinen gesteuert wird (siehe $>$ Tab. 1). Die aufgeführten Reaktionsweisen und Zytokinaktivierungen stellen vermutlich nur einen Teil des komplexen Geschehens dar. Ähnliche Vorgänge finden sich auch bei anderen entzündlichen Erkrankungen der Haut, führen aber zu etwas anderen klinischen Veränderungen. Deutlich wird das bei der atopischen Dermatitis, die anfangs durch Rötung, Schuppung und Juckreiz gekennzeichnet ist, langfristig aber auch zu Lichenifikation und Pachydermie führen kann.
Die klinische Wirkung der KPE ist seit langem nachgewiesen und reduziert mit dem Ödem auch die Inflammation und damit die Umwandlung des Gewebes. Die genauen Kenntnisse dieser Abläufe sind der Schlüssel für eine systemische Therapie, die damit auch ein Meilenstein in der Behandlung bedeuten würde. Gezielte Antikörperbehandlungen, wie sie erfolgreich bei anderen entzündlichen Erkrankungen, wie der rheumatoiden Arthritis oder Psoriasis, eingesetzt werden, haben bisher in keiner klinischen Studie signifikante Besserung gezeigt. LTB4-Hemmung durch Ketoprofen wurde bisher vor allem am Mausmodell untersucht. Hydroxytyrosol zeigt in vitro gute Erfolge und Tacrolimus wurde an niedrigen Fallzahlen bei plastischen Operationen angewendet. Dennoch sind die Ansätze vielversprechend und lymphologische 
Forschung auf diesem Gebiet ist dringend erforderlich. Ein Schritt aus klinischer Sicht wäre die Betrachtung von Patienten mit Lymphödemen, die gleichzeitig wegen einer anderen Erkrankung immunsuppressiv behandelt werden. Findet sich dort eine geringere Fibrosierung?

Die weitere Entschlüsselung der Vorgänge im Gewebe ist der wichtigste nächste Schritt in der Lymphologie.

Interessenkonflikt

Die Autorinnen/Autoren geben an, dass kein Interessenkonflikt besteht.

Literatur

[1] Gjorup CA, Groenvold M, Hendel HW et al. Health-related quality of life in melanoma patients: impact of melanoma-related limb lymphoedema. Eur J Cancer 2017; 85: 122-132 https://doi.org/10.1016/j. ejca.2017.07.052

[2] Kataru RP, Wiser I, Baik JE et al. Fibrosis and secondary lymphedema: chicken or egg? Translational Research 2019; 209: 6876

[3] Ly CL, Kataru RP, Mehrara BJ. Inflammatory manifestations of lymphedema. Int J Mol Sci 2017; 18: doi:10.3390/ijms18010171

[4] Ly CL, Nores GDG, Kataru RP et al. T helper 2 differentiation is necessary for development of lymphedema. Transl Res 2019; 206: 57-70 https:/ doi.org/10.1016/j.trsl.2018.12.003

[5] Karlsen TV, Karkkainen M], Alitalo K et al. Transcapillary fluid balance consequences of missing initial lymphatics studied in a mouse model of primary lymphoedema. J Physiol 2006; 574: 583-596

[6] Cuzzone DA, Weitman ES, Albano NJ et al. IL-6 regulates adipose deposition and homeostasis in lymphedema. Am J Physiol Heart Circ Physiol 2014; 306: H1426-H1434

[7] Liao S, Cheng G, Conner DA et al. Impaired lymphatic contraction associated with immunosuppression. Proc Natl Acad Sci USA 2011; 108 : 18784-18789
[8] Angeli V, Randolph G]. Inflammation, lymphatic function, and dendritic cell migration. Lymphat Res Biol 2006; 4: 217-228

[9] Tian W, Rockson SG, Jiang X et al. Leukotriene B4 antagonism ameliorates experimental lymphedema. Sci Transl Med 2017; 9: eaal3920. doi:10.1126/scitranslmed.aal3920

[10] Gordon K, Schulte D, Brice G et al. Mutation in vascular endothelial growth factor-c, a ligand for vascular endothelial growth factor receptor-3, is associated with autosomal dominant milroy-like primary lymphedema. Circ Res 2013; 112: 956-960 https://doi.org/10.1161/CIR CRESAHA.113.300350

[11] Jiang $X$, Nicolls MR, Tian W et al. Lymphatic dysfunction, leukotrienes, and lymphedema. Annu Rev Physiol 2018; 80: 49-70 https://doi.org/ 10.1146/annurev-physiol-022516-034008

[12] Avraham T, Zampell JC, Yan A et al. Th2 differentiation is necessary for soft tissue fibrosis and lymphatic dysfunction resulting from lymphedema. FASEB ] 2013; 27: 1114-1126 https://doi.org/10.1096/fj.12222695

[13] Savetsky IL, Ghanta S, Gardenier JC et al. Th2 cytokines inhibit lymphangiogenesis. PLoS One 2015; 10: e0126908 https://doi.org/10.1371/jour nal.pone.0126908

[14] Zampell JC, Yan A, Elhadad S et al. CD4+ cells regulate fibrosis and lymphangiogenesis in response to lymphatic fluid stasis. PLoS One 2012; 7: e49940 https://doi.org/10.1371/journal.pone.0049940

[15] Rockson SG, Tian W, Jiang X et al. Pilot studies demonastrate the potential benefits of anti-inflammatory therapy in human lymphedema. $\mathrm{JCl}$ insight 2018. doi:10.1172/jci.insight. 123775

[16] Bertelli M, Kiani AK, Paolacci S et al. Molecular pathways involved in lymphedema: Hydroxytyrosol as a candidate natural compound for trating the effects of lymph accumulation. Journal of Biotechnology 2020; 308: 82-86

[17] Van, Y-Vu Robert, Wald G, Lu C et al. The Effect of Topical Tacrolimus on Pedicled Flap Survival. Ann Plast Surg 2020; 85 (Suppl. 1): S118-S121. doi:10.1097/SAP.000000000000233 